ఠ

\title{
O-GlcNAcylation enhances the invasion of thyroid anaplastic cancer cells partially by PI3K/Akt I pathway
}

\section{Peng Zhang \\ Chunli Wang \\ Tao Ma \\ Shengyi You}

General Surgery Department, General Hospital of Tianjin Medical University, Tianjin, People's Republic of China
Correspondence: Shengyi You General Surgery Department, General Hospital of Tianjin Medical University, Tianjin 300050,

People's Republic of China

Tel +862260363745

Email yshengyi2000@163.com
This article was published in the following Dove Press journal:

OncoTargets and Therapy

9 November 2015

Number of times this article has been viewed

Background: The PI3K family participates in multiple signaling pathways to regulate cellular functions. PI3K/Akt signaling pathway plays an important role in tumorigenesis and development. $O$-GlcNAcylation, a posttranslational modification, is thought to modulate a wide range of biological processes, such as transcription, cell growth, signal transduction, and cell motility. $O$-GlcNAcylation is catalyzed by the nucleocytoplasmic enzymes, OGT and OGA, which adds or removes $O$-GlcNAc moieties, respectively. Abnormal $O$-GlcNAcylation has been implicated in a variety of human diseases. However, the role of $O$-GlcNAcylation in tumorigenesis and progression of cancer is still under-investigated. Understanding the $O$-GlcNAc-associated molecular mechanism might be significant for diagnosis and therapy of cancer.

Methods: Human thyroid anaplastic cancer $8305 \mathrm{C}$ cells were used to evaluate the role of $O$-GlcNAcylation in tumorigenesis and progression of cancer. The global $O$-GlcNAc level of intracellular proteins was up-regulated by OGA inhibitor Thiamet-G treatment or OGT overexpression. Cell proliferation was assessed by MTT assay. Invasion in vitro was determined by Transwell assay, and phosphorylation of Akt1 at Ser473 was assessed by Western blot for activity of Akt1. PI3K-specific inhibitor LY294002 and RNA interference of Akt1 were used to investigate the impact of PI3K/Akt signaling on the regulation of $O$-GlcNAcylation during tumor progression.

Results: Cell models with remarkably up-regulated $O$-GlcNAcylation were constructed, and then cell proliferation and invasion were determined. The results indicated that the proliferation was not affected by OGA inhibition or OGT overexpression, while the invasion of $8305 \mathrm{C}$ cells with OGA inhibition or OGT overexpression was obviously increased. Akt1 activity was stimulated by elevated $O$-GlcNAcylation by mediating phosphorylation at Ser473. The enhanced invasion of thyroid cancer cells by Thiamet-G treatment or OGT overexpression was significantly depressed by PI3K inhibitor LY294002. Moreover, silence of Akt1 remarkably attenuated the increase of cell invasion induced by Thiamet-G treatment, but the invasion was still higher compared to Akt1-silenced only cells. In other words, Thiamet-G restored the invasion of Akt1-silenced thyroid cancer cells, but it was still lower relative to Thiamet-G-treated only cells.

Conclusion: Taken together, our findings suggested that $O$-GlcNAcylation enhanced the invasion of thyroid anaplastic cancer cells partially by PI3K/Akt signaling, which might be a potential target for the diagnosis and treatment of thyroid anaplastic cancer.

Keywords: $O$-GlcNAcylation, thyroid anaplastic cancer, invasion, PI3K/Akt, Akt1

\section{Introduction}

The PI3K family participates in multiple signaling pathways to regulate cellular functions. The lipid products produced by PI3K activation, $\mathrm{PI}(3,4) \mathrm{P} 2$ and $\mathrm{PI}(3,4,5) \mathrm{P} 3$ as the second messengers, bind and activate the intracellular target proteins to form a 
signal transduction cascade, and finally adjust proliferation, differentiation, survival, and migration of cells. ${ }^{1}$ Akt (or $\mathrm{PKB})$ is a serine/threonine protein kinase, the downstream molecule of PI3K. There are at least three Akt family members: Aktl/PKB $\alpha, A k t 2 / P K B \beta$, and Akt3/PKB $\gamma$, which play individual roles respectively in the regulation of cell functions. PI3K/Akt signaling pathway plays a significant role in tumorigenesis and development. In recent years, the signal transduction pathway has attracted a great deal of attention and has become an important target for cancer treatment.

There are two ways to activate PI3K. First, interaction with growth factor receptor with phosphorylated tyrosine residues or junction protein leads to PI3K activation by change of the dimer conformation. Second, direct combination with Ras and P110 contributes to activation of PI3K. ${ }^{2}$ PI3K activation generates the second messenger PIP3 in plasma membrane interplay with signaling protein Akt and PDK1 containing PH structural domain, promoting the activation of Akt by Thr308 phosphorylation by PDK1. Akt could also be activated by the phosphorylation of Ser473 induced by PDK2 (such as ILK). ${ }^{3}$ Activated Akt activates or inhibits the downstream target proteins, such as Bad, caspase-9, NFкB, GSK-3, FKHR, p21Cip1, and p27 Kip1, and further regulates cell proliferation, differentiation, apoptosis, and migration.

Invasion is a critical process during tumor metastasis. PI3K can deliver integrin-mediated invasion signal, necessary especially for integrin $\alpha 2 \beta 1$-, $\alpha 6 \beta 4$-, and $\alpha \mathrm{V} \beta 3$ - mediated invasion behavior. For example, PI3K- $\alpha$ V $\beta 3$-mediated invasion is a characteristic of prostate cancer. In breast cancer and ovarian cancer, overexpression of Akt 2 could up-regulate integrin $\beta 1$ through Col4 to increase cell invasion and metastasis. ${ }^{4}$ Sustained expression of Akt could induce epithelial mesenchymal transition of squamous cancer cell lines to enhance cellular motility needed in tissue invasiveness and metastasis..$^{5}$ These results implied that PI3K/Akt signaling had a critical impact on tumor cell invasion.

$O$-GlcNAcylation, a posttranslational modification of serine and threonine groups on nuclear and cytoplasmic proteins with $O$-GlcNAc, is thought to modulate the function and activity of proteins in cells. ${ }^{6}$ Reversible $O$-GlcNAcylation is catalyzed by the nucleocytoplasmic enzymes, OGT and OGA, which adds or removes $O$-GlcNAc moieties, respectively. ${ }^{7}$ $O$-GlcNAcylation is involved in a wide range of biological processes, such as transcription, cell growth, signal transduction, cell motility, and metabolism. ${ }^{8-10}$ Abnormally regulated $O$-GlcNAcylation has been implicated in diseases such as diabetes, Alzheimer's disease, and cancer. ${ }^{7,11}$ However, the role of $O$-GlcNAcylation in tumorigenesis and progression of cancer is still under-investigated.

In this study, we investigated whether increased $O$-GlcNAcylation of cellular proteins could affect PI3K/ Akt signaling in $8305 \mathrm{C}$ thyroid anaplastic cancer cells. Our results demonstrated, for the first time, that up-regulation of $O$-GlcNAcylation enhances the invasion of thyroid cancer cells partially by promoting PI3K/Akt1 signaling.

\section{Material and methods Cell culture and treatments}

The $8305 \mathrm{C}$ thyroid anaplastic cancer cells (European Collection of Cell Cultures), were grown in advanced minimum essential medium supplemented with $2 \mathrm{mM}$ glutamine and $5 \%$ fetal bovine serum in a humidified atmosphere containing $5 \% \mathrm{CO}_{2}$ at $37^{\circ} \mathrm{C}$. Cells were treated with $5 \mu \mathrm{M}$ of the OGA inhibitor Thiamet-G (Thia-G) for 24 hours or the indicated time period. When grown to $80 \%$ fusion, $8305 \mathrm{C}$ cells were cultured in medium without serum (if treating with Thia-G, $5 \mu \mathrm{M}$ of Thia-G should be added at this time). Cells were treated with $2 \mu \mathrm{M}$ of LY294002 for 24 hours (control was treated with the same volume of dimethyl sulfoxide) to be used for immunoblotting or were counted and prepared for invasion assay.

pCMV-Flag-OGT, a Flag-tagged human nuclear/ cytoplasmic OGT expression vector was used. ${ }^{12}$ Cell transfections were carried out using Lipofectamine ${ }^{\mathrm{TM}} 2000$ (Thermo Fisher Scientific, Waltham, MA, USA) according to the manufacturer's instructions.

Small interfering RNA (siRNA) that targets the coding region of human Akt1 messenger RNA (mRNA) sequences was designed and used to suppress Akt1 expression in the $8305 \mathrm{C}$ cells. The targeting sequences were: siAkt1-1, GCTACTTCCTCCTCAAGAACG; siAkt1-2, GGACGGGCACATCAAGATAAC; control(Ctrl), ACGTGACACGTTCGGAGAATT. Small interfering Akt1 sequences were cloned into pLKO.1 lentivirus vectors. Lentiviruses of pLKO.1-based vectors were produced by co-transfection with $\Delta 8.2$ and VSV-G plasmids into HEK293T cells. The infected cells were treated with $8 \mu \mathrm{g} / \mathrm{mL}$ puromycin for 2 weeks.

\section{Real-time reverse transcription polymerase chain reaction (RT-PCR)}

RNA was isolated from the $8305 \mathrm{C}$ cells using the Total RNA Isolation kit (A\&A Biotechnology, Gdynia, Poland) according to the manufacturer's instructions. First strand cDNAs were obtained by reverse transcription of $1 \mu \mathrm{g}$ of total RNA 
using RevertAid ${ }^{\mathrm{TM}}$ First Strand cDNA Synthesis Kit (Thermo Fisher Scientific) following the manufacturer's protocol. Real-time amplification of the cDNA was performed using TaqMan $^{\circledR}$ Gene Expression Assay (Thermo Fisher Scientific) according to the manufacturer's instructions. The fluorogenic, FAM-labeled probes and the sequence-specific primers of gene coding Akt1 and the internal control glyceraldehyde 3-phosphate dehydrogenase (GAPDH) were used. Fold differences in Akt1 expression, normalized to GAPDH levels were calculated using the formula $2^{\Delta \Delta \mathrm{Ct}}$. Relative amounts of mRNA in siRNA-treated cells are indicated as the percentage of the amount of mRNA in the untreated cells.

\section{Western blotting}

Cells were lysed in cell lysis buffer for Western blot and IP (P0013, Beyotime Biotechnology, Jiangsu, People's Republic of China) containing a protease inhibitor cocktail (Hoffman-La Roche Ltd., Basel, Switzerland) and $5 \mu \mathrm{M}$ PUGNAc (an OGA inhibitor; Toronto Research Chemicals Inc., Toronto, Canada). Protein samples $(50 \mu \mathrm{g})$ were separated by $12 \%$ sodium dodecyl sulfate polyacrylamide gel electrophoresis (SDS-PAGE) and transferred to Immobilon-P membranes (EMD Millipore, Billerica, MA, USA). Antibodies to $O$-GlcNAc (RL2, Affinity Bio-Reagents, Golden, CO, USA; CTD110.6, Abcam plc, Cambridge, UK), OGT (F-12; Santa Cruz, CA, USA), Akt1 (Cell Signaling Technology, Beverly, MA, USA), phospho-Akt1 (Ser473, Cell Signaling Technology), and GAPDH (Santa Cruz, CA, USA) were used for detection by ECL detection reagent (GE Healthcare UK Ltd, Little Chalfont, UK).

\section{Cell proliferation assay}

Cell proliferation was assessed by MTT dye conversion. Briefly, cells were seeded $10^{4}$ per well in a 96-well, flat bottom plate after transfection. Cells were allowed to grow in a $37^{\circ} \mathrm{C}, 5 \% \mathrm{CO}_{2}$ incubator for 48 hours, followed by another 4 hours after $20 \mu \mathrm{L}$ of $5 \mathrm{mg} / \mathrm{mL}$ MTT was added to each well. Cells were lysed by addition of $200 \mu \mathrm{L}$ dimethyl sulfoxide. Absorbance was measured at $490 \mathrm{~nm}$ using an enzyme-linked immunosorbent assay (ELISA) reader.

\section{Cell invasion assay}

Invasion assay was performed using Transwell chambers (6.5 mm; Corning Incorporated, Corning, NY, USA) with $8 \mu \mathrm{m}$ pore membranes. The upper face of the membrane was covered with $70 \mu \mathrm{L}$ of Matrigel $(1 \mathrm{mg} / \mathrm{mL}$; BD Biosciences, San Jose, CA, USA). The lower chamber was filled with $600 \mu \mathrm{L}$ of NIH-3T3-conditioned medium with or without
OGA inhibitors. Cells $\left(5 \times 10^{4}\right)$ were suspended with $100 \mu \mathrm{L}$ of advanced MEM with $1 \%$ fetal calf serum and plated into the upper chamber with or without OGA inhibitors. After 24 hours, the number of cells appearing by crystal violet staining on the undersurface of the polycarbonate membranes was scored visually in six random fields at $100 \times$ magnification using a light microscope.

\section{Statistical analysis}

All of the experiments were repeated at least three times. Statistical significance was analyzed using the SPSS 11.0 software program. The value of $P<0.05$ was considered statistically significant. Data were presented as the mean \pm standard error of the mean.

\section{Results}

\section{Up-regulation of $O$-GlcNAcylation level}

To increase the $O$-GlcNAcylation of proteins, $8305 \mathrm{C}$ cells were treated with Thia-G which is a highly selective OGA inhibitor that potently inhibits activity of OGA in cells. Thia-G, by preventing $O$-GlcNAc cycling of proteins, leads to globally elevated levels of this modification. The $8305 \mathrm{C}$ cells were treated with $5 \mu \mathrm{M}$ Thia-G for 24 hours. As expected, Thia-G effectively increased the $O$-GlcNAc level in treated cells (Figure 1A). Additionally, we used the alternative methodological approach to up-regulate $O$-GlcNAc level, ie, OGT protein overexpression. Human nuclear/ cytoplasmic OGT expression plasmid (500 ng) was used to transfect into $8305 \mathrm{C}$ cells for 24 hours. The result showed that OGT overexpression drastically elevated OGT protein expression as well as $O$-GlcNAcylation level in $8305 \mathrm{C}$ cells (Figure 1B). These approaches enabled us to identify the effects of $O$-GlcNAcylation on the biological properties of thyroid anaplastic cancer cells.

\section{O-GlcNAcylation does not affect the proliferation of thyroid anaplastic cancer cells}

To investigate whether $O$-GlcNAcylation affects cell proliferation, $8305 \mathrm{C}$ cells were treated with $5 \mu \mathrm{M}$ Thia-G or $500 \mathrm{ng}$ OGT plasmid transfection for 24 hours. MTT assay was performed. Surprisingly, Both OGA inhibition and OGT overexpression did not affect cellular growth (Figure 2A and B).

\section{O-GlcNAcylation enhances the invasion of thyroid anaplastic cancer cells}

To determine whether the changes in $O$-GlcNAcylation level affect cell invasion in vitro, thyroid anaplastic cancer 8305C 

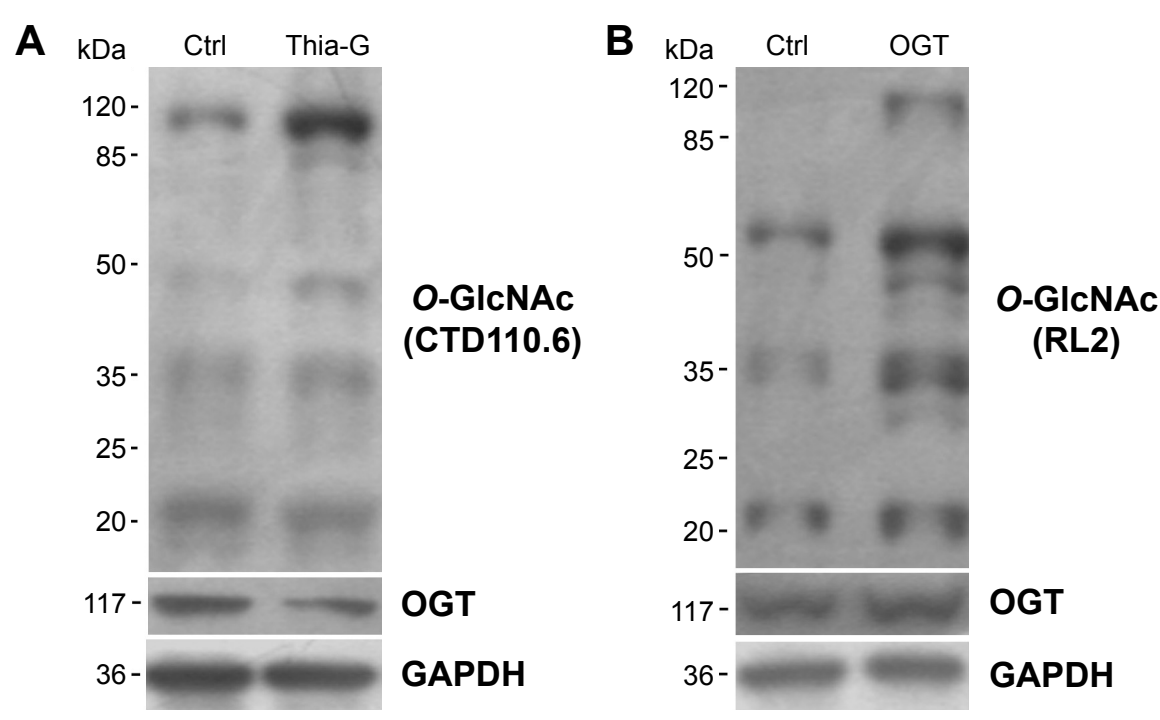

Figure I O-GIcNAcylation was up-regulated by Thiamet-G treatment and OGT overexpression.

Notes: The $8305 \mathrm{C}$ cells were treated with $5 \mu \mathrm{M}$ Thiamet-G (Thia-G) (A) or OGT overexpression (B) for 24 hours. Western blot was used for analysis of changes in the O-GlcNAcylation level of cellular proteins with anti-O-GlcNAc antibodies (CTDI I0.6 and RL2). Anti-OGT (F-I2) antibody was used to detect OGT protein expression.

Abbreviations: Ctrl, control; GAPDH, glyceraldehyde 3-phosphate dehydrogenase.

cells were treated with $5 \mu \mathrm{M}$ Thia-G or $500 \mathrm{ng}$ OGT plasmid transfection for 24 hours. Then invasion assay was carried out. The result indicated that both OGA inhibition and OGT overexpression markedly enhanced cell invasion relative to the untreated or OGT-unexpressed controls (Figure 3A and B). The aforementioned results suggested that the increased invasion mediated by $O$-GlcNAcylation was irrelevant to cell proliferation.

\section{Up-regulation of O-GlcNAcylation} increases Akt I phosphorylation at Ser473 The $8305 \mathrm{C}$ cells were treated with $5 \mu \mathrm{M}$ Thia-G or were transfected with $500 \mathrm{ng}$ OGT plasmid, and then cell lysates were resolved by SDS-PAGE, and phospho-Akt1 (Ser473) or Akt1 were determined with specific antibodies (Figure 4A and B).
An increase in Ser473 phosphorylation was noted both in the cells treated with Thia-G and OGT plasmid in comparison to the control cells (untreated or treated with empty vector). This result suggested that increased $O$-GlcNAcylation promoted invasion of thyroid cancer cells probably by stimulating Aktl activation.

\section{LY294002 depresses the promotive effect of $\mathrm{O}$-GlcNAcylation on invasion of thyroid anaplastic cancer cells}

Considering that Akt1 activity could be strictly controlled by the upstream protein PI3K, we speculated that PI3K/Akt signaling pathway might play a role in the modulation of $O$-GlcNAcylation in invasion of thyroid anaplastic cancer cells. Therefore, PI3K-specific inhibitor LY294002 was used
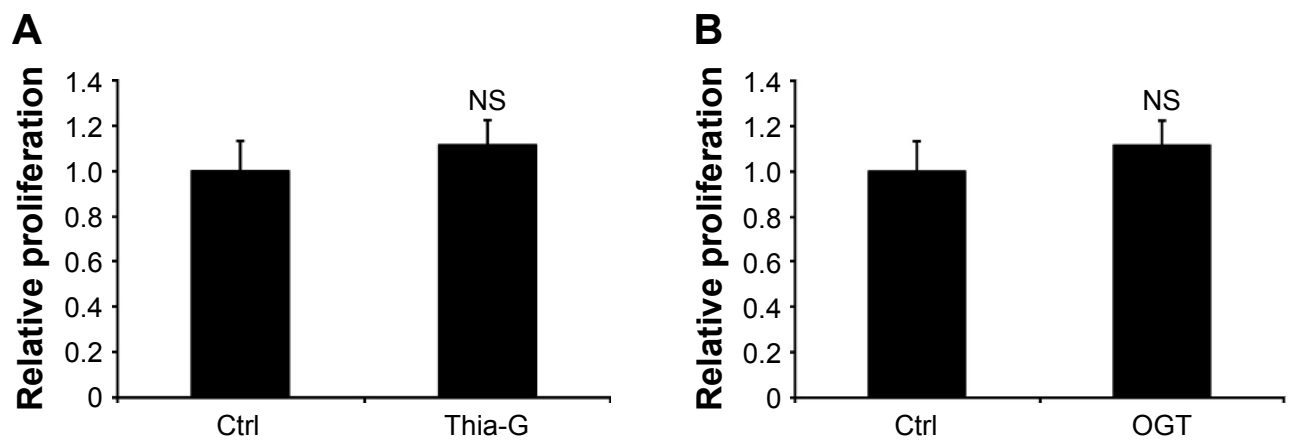

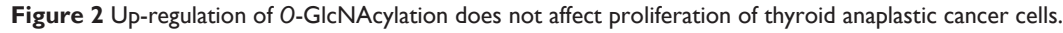

Notes: MTT assay was used to evaluate cell proliferation. (A) Thiamet-G (Thia-G) treatment and (B) OGT overexpression did not significantly increase the proliferation of $8305 \mathrm{C}$ cells.

Abbreviations: NS, no significance; Ctrl, control. 

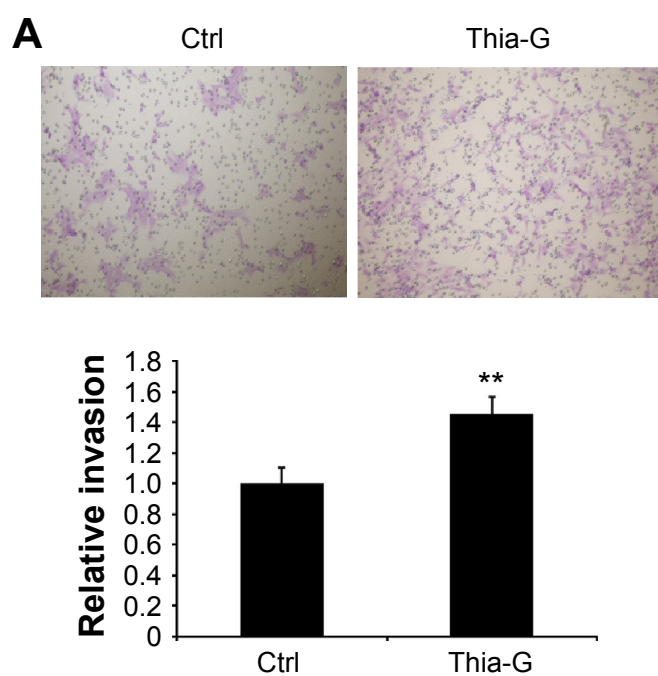

B Ctrl OGT
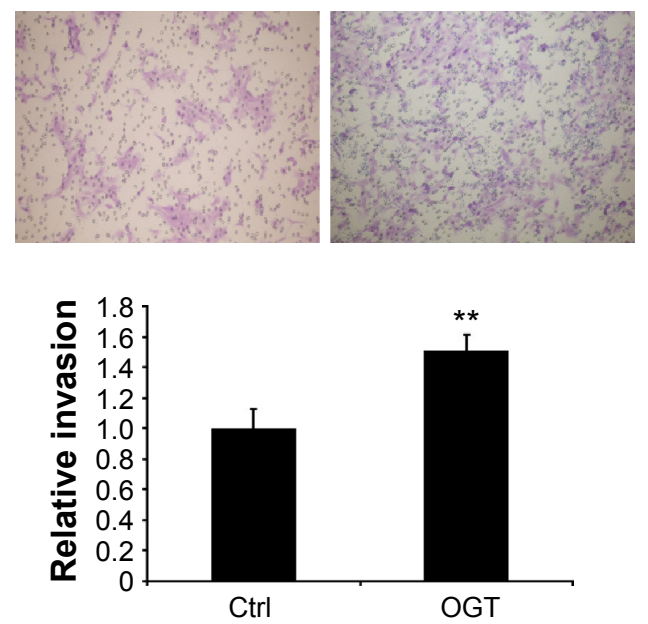

Figure 3 Up-regulation of $O$-GlcNAcylation enhances the invasion of thyroid anaplastic cancer cells in vitro.

Notes: Transwell assay was used to assess the invasion of thyroid anaplastic cancer cells. (A) Thiamet-G (Thia-G) treatment and (B) OGT overexpression significantly promoted the invasion of $8305 \mathrm{C}$ cells. ${ }^{* * P}<0.0$ I (Student's $t$-test). Error bars represent the mean \pm standard error of the mean of three independent experiments.

Abbreviation: Ctrl, control.

to block PI3K/Akt pathway to determine the aforementioned hypothesis. First, the impact of LY294002 on Akt1 activity in 8305 C cells was determined. It was shown that LY294002 notably decreased phosphorylation at Ser473 of Akt1 (Figure 5A). Based on the result, invasion assay of thyroid anaplastic cancer cells was performed (Figure 5B). We demonstrated that LY294002 significantly attenuated the promotive impact of $O$-GlcNAcylation on invasion of thyroid anaplastic cancer cells accompanied by reduced Aktl activity, but the invasion was still higher than that of untreated control cells. It was implied that PI3K/Akt signaling was involved in the regulation of $O$-GlcNAcylation in invasion of thyroid cancer cells, but was not an exclusive approach.

\section{Silence of Akt I protein in thyroid anaplastic cancer cells}

To verify the aforementioned results, two siRNA duplexes (siAkt1-1 and siAlt1-2) were used to down-regulate the
A
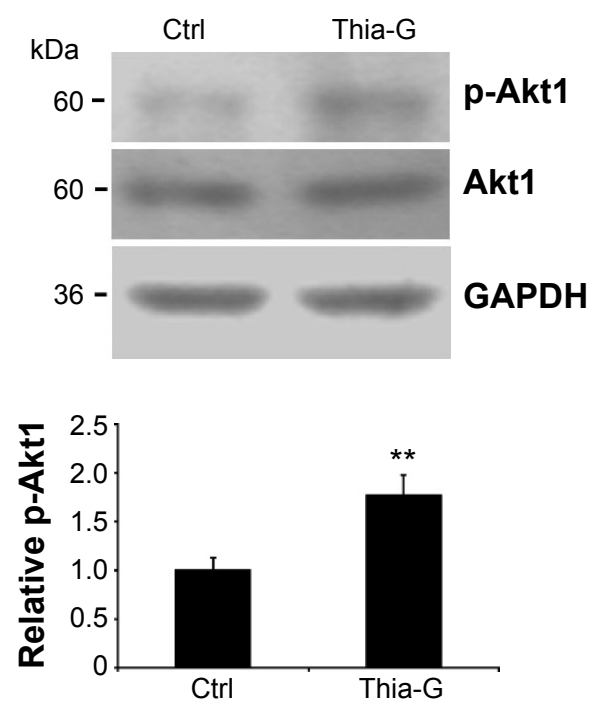

B
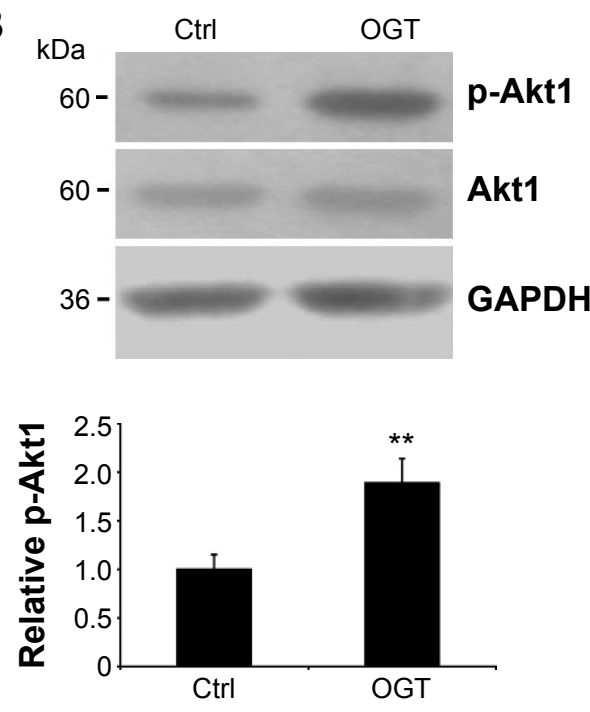

Figure $4 \mathrm{Up}$-regulation of $\mathrm{O}$-GlcNAcylation increases Akt l phosphorylation.

Notes: The $8305 \mathrm{C}$ cells were treated with (A) $5 \mu \mathrm{M}$ Thiamet-G (Thia-G) or (B) OGT overexpression for 24 hours. Cell lysates were prepared and used for Western blots for p-Aktl (Ser473), Aktl, and GAPDH (loading control) with anti-p-Aktl (Ser473), anti-Aktl, and anti-GAPDH antibodies. The blots of p-Aktl were quantified by densitometry, and the results were expressed as a ratio relative to the values obtained in untreated control cells. Error bars represent the mean \pm standard error of the mean of three independent experiments, $* * P<0.01$.

Abbreviations: Ctrl, control; GAPDH, glyceraldehyde 3-phosphate dehydrogenase. 

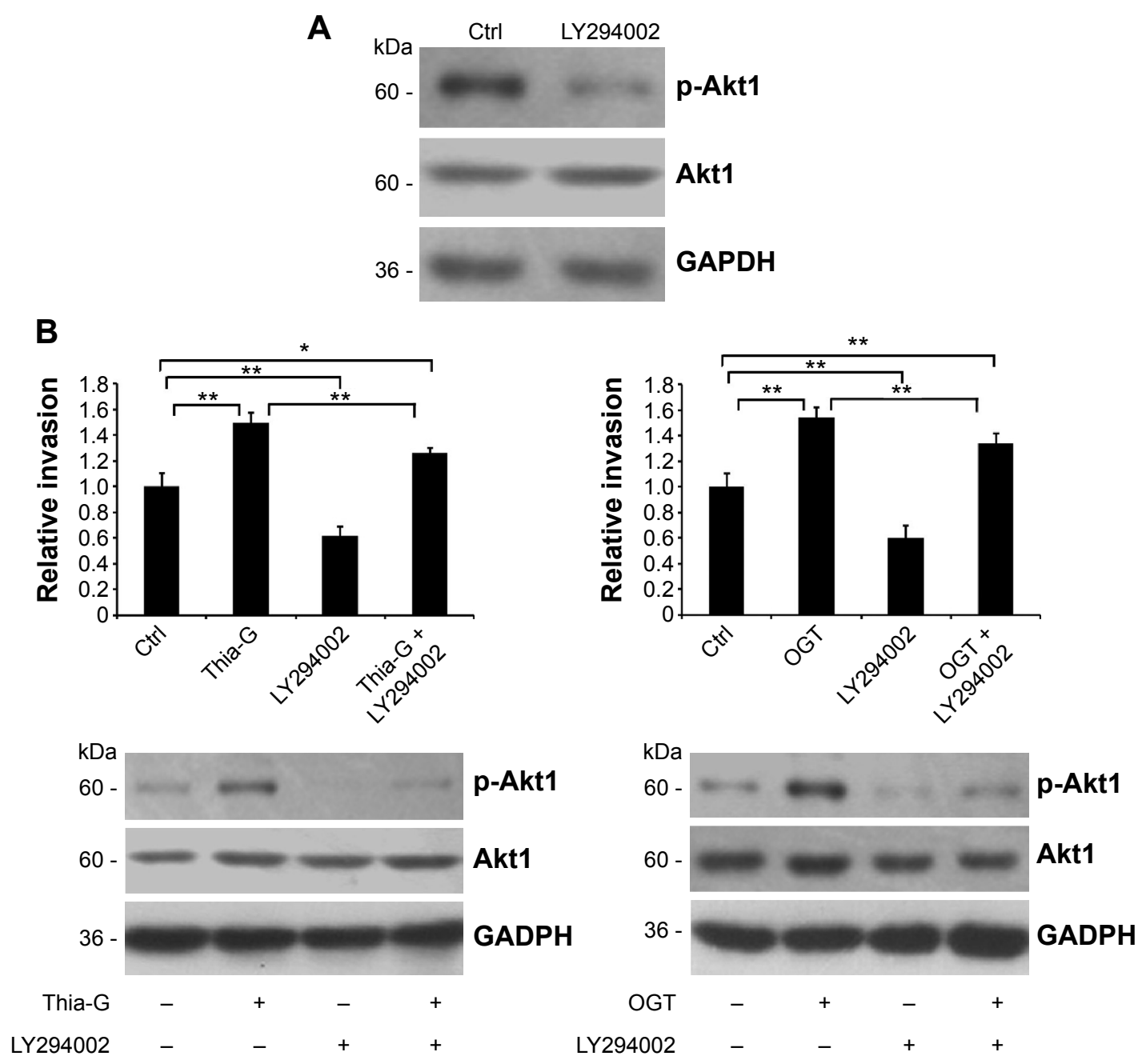

Figure 5 The inhibition of LY294002 on the regulation of O-GlcNAcylation on invasion of thyroid anaplastic cancer cells.

Notes: For 24 hours, $5 \mu$ M Thiamet-G (Thia-G) treated or untreated and OGT overexpression or underexpressed $8305 \mathrm{C}$ cells were treated with $2 \mu \mathrm{M}$ LY294002. (A) The inhibition of LY294002, a specific inhibitor of PI3K, on Aktl phosphorylation (Ser473) was assessed by Western blot. (B) Transwell assay analyzed the impact of LY294002 on invasion of Thiamet-G treated (on the left) or OGT overexpression (on the right) in $8305 \mathrm{C}$ cells. Western blot analysis showed the corresponding phosphorylation (Ser473) of Aktl, demonstrating the changes of Aktl activity in respectively treated cells with Thiamet-G, OGT overexpression or LY294002. Anti-p-Akt (Ser473), anti$\mathrm{Aktl}$, and anti-GAPDH antibodies were used in the experiments. Error bars represent the mean \pm standard error of the mean of three independent experiments, $* P<0.05$, $* * P<0.01$.

Abbreviations: Ctrl, control; GAPDH, glyceraldehyde 3-phosphate dehydrogenase.

expression of Akt1. The control cells were transfected with a non-silent scrambled RNA duplex. The effect of RNAi was assessed by real-time RT-PCR and Western blot analysis. Both siRNAs caused an obvious decrease in the mRNA level and protein expression (Figure 6A and B). However, siAkt1-2 was more effective in reduction of Akt1 level, thus it was chosen for further experiments.

\section{Akt I silencing disrupts the promotion of $O-G l c N A c y l a t i o n$ on invasion of thyroid anaplastic cancer cells}

Akt1-silenced $8305 \mathrm{C}$ cells were treated with $5 \mu \mathrm{M}$ Thia-G to assess the impact of Akt1 on the process that
$O$-GlcNAcylation enhanced the invasion of thyroid cancer cells. The results revealed that Akt 1 silencing remarkably attenuated the increase of cell invasion induced by Thia-G treatment, but the invasion was still higher compared to Akt1-silenced only cells. In other words, Thia-G restored the invasion of Akt1-silenced thyroid cancer cells, but it was still lower relative to Thia-G only-treated cells and higher relative to untreated control cells (Figure 7A). Western blotting showed the levels of Akt1 protein and its Ser473 phosphorylation in cells (Figure 7B). These findings further suggested that $O$-GlcNAcylation enhanced the invasion of thyroid anaplastic cancer cells partially by stimulating Akt1 activation. 


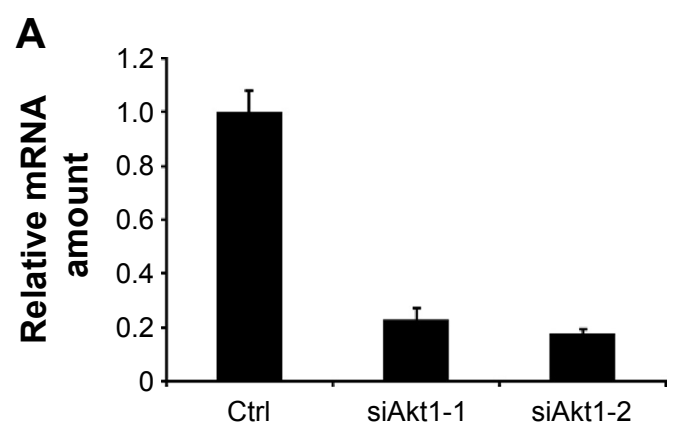

B

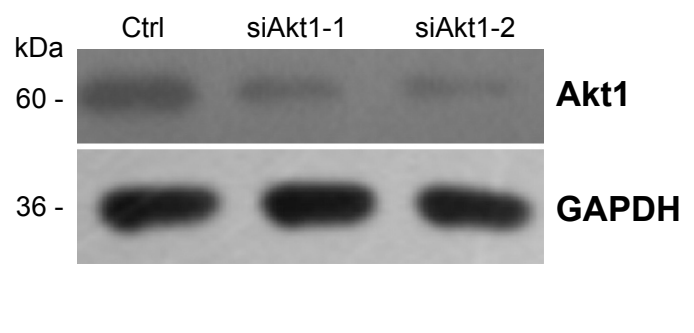

Figure 6 RNAi-mediated down-regulation of Aktl.

Notes: The $8305 \mathrm{C}$ cells were treated with two different small interfering RNA duplexes (siAktl-I and siAkt l-2) or control (Ctrl) scrambled siRNA. (A) Relative amount of Akt l mRNA 48 hours after cells were transfected with $30 \mathrm{nM}$ siRNAs (siAktl-I, siAktl-2) as assessed by real-time RT-PCR. (B) Western blot analysis of changes in Akt I protein expression level in lysates of $8305 \mathrm{C}$ cells treated with $30 \mathrm{nM}$ siRNAs for 48 hours. Western blot analysis was performed with Aktl and GAPDH antibodies.

Abbreviations: RT-PCR, reverse transcription polymerase chain reaction; mRNA, messenger RNA; GAPDH, glyceraldehyde 3-phosphate dehydrogenase; Ctrl, control.

\section{Discussion}

$O$-GlcNAcylation, a regulatory posttranslational modification more analogous to phosphorylation, widely occurs in nuclear and cytoplasmic proteins. $O$-GlcNAcylation is implicated in some important cellular processes such as regulation of gene expression, protein proteolysis, and signal transduction. ${ }^{8-10}$ Recent research has shown that $O$-GlcNAcylation is closely related to a variety of human cancers. ${ }^{13-15}$ In this study, we up-regulated $O$-GlcNAcylation level in thyroid anaplastic cancer cells by Thia-G treatment or overexpression of OGT and found that up-regulation of $O$-GlcNAcylation obviously enhanced cell invasion. $\mathrm{PI} 3 \mathrm{~K} /$ Akt signaling pathway is associated with a variety of malignant human tumors, such as breast cancer, lung cancer, melanoma, and lymphoma ${ }^{16}$ and is thought to function in a wide range of biological processes, eg, cell adhesion, growth, migration, invasion, and angiogenesis. ${ }^{1,417-21}$ Akt functions in the anti-apoptosis pathway by phosphorylation of downstream target protein. Sustained activation of Akt could prevent cell apoptosis mediated by PTEN by phosphorylating Bcl-2 family members BAD and protease caspase-9; Akt could phosphorylate $\mathrm{\kappa B}$ kinase to accelerate

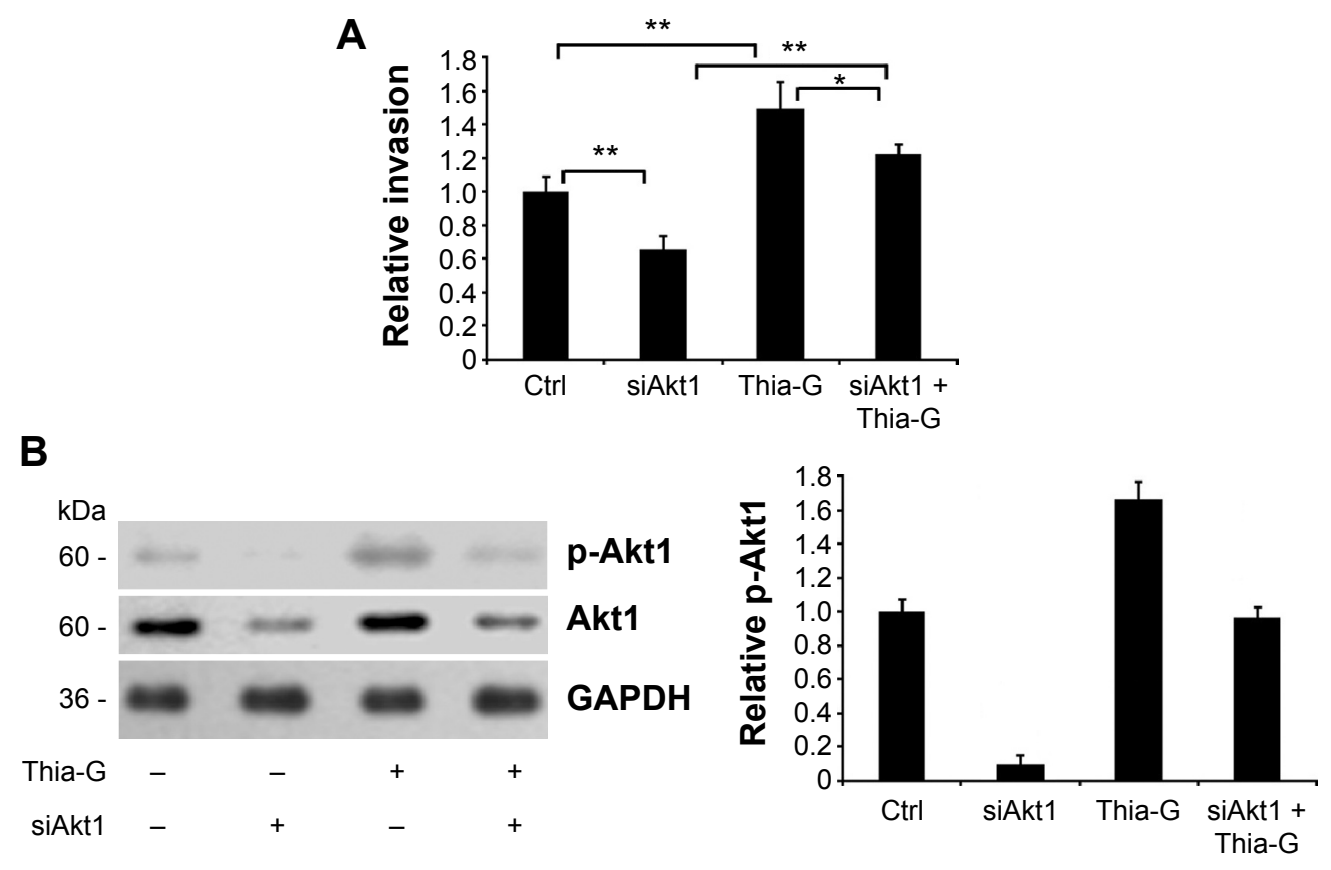

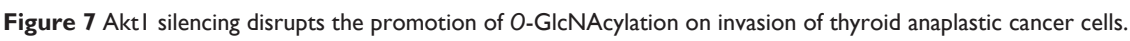

Notes: (A) Akt l-silenced or -non-silenced $8305 \mathrm{C}$ cells were treated or untreated with $5 \mu \mathrm{M}$ Thiamet-G (Thia-G) for 24 hours. Invasion assay in vitro was carried out. Error bars represent the mean \pm standard error of the mean of three independent experiments, $* P<0.05$, $* * P<0.01$. (B) Protein expression of Akt l and its phosphorylation in cells was evaluated by Western blotting using anti-AktI and anti-pSer473-AktI antibodies. These blots are representative of three independent experiments. The blots were quantified by densitometry, and the results were expressed as a ratio relative to the values obtained in untreated control (Ctrl) cells. 
$\kappa \mathrm{B}$ degradation leading to nuclear translocation of $\mathrm{NF} \kappa \mathrm{B}$ to further activate the target genes for cell survival. ${ }^{18}$ Additionally, Akt affected cell proliferation through regulation of cell cycle. Akt inhibited GSK3 kinase activity by direct phosphorylation to prevent the degradation of cyclin D1; Akt could also negatively regulate the expression of CKIs, such as p27Kip1 and p21Cip1. Akt modulated the activity of p21Cip1 by influencing the phosphorylation level and the binding with PCNA to increase cell proliferaion; ${ }^{17} \mathrm{Akt}$ could also directly phosphorylate $\mathrm{p} 27 \mathrm{Kip} 1$ at Thr157 resulting in $\mathrm{p} 27 \mathrm{Kip} 1$ retention in the cytoplasm, preventing cell cycle blockade mediated by $\mathrm{p} 27 \mathrm{Kip} 1 .^{22}$ However, in this study, we have not found a difference in $O$-GlcNAcylationinduced proliferation by Akt activation in thyroid anaplastic cancer $8305 \mathrm{C}$ cells. This result indicates that the biological effects of Akt on cell survival and growth might be celltype specific.

Besides, PI3K/Akt signaling involved in the tumor angiogenesis process is mediated by multiple factors and could deliver invasion and metastasis signals by multiple pathways. PI3K participated in endothelial signaling mediated by vascular endothelial growth factor (VEGF) by interacting with E-cadherin, $\beta$-catenin, and vascular endothelial growth factor receptor (VEGFR)-2 to stimulate $\mathrm{PI} 3 \mathrm{~K} / \mathrm{Akt}$ pathway, and the complex of VEGFR-2- and $\alpha \mathrm{V} \beta 3$-mediated endothelial cell adhesion and migration also by the PI3K-dependent way; ${ }^{1,20,21}$ $\mathrm{PI} 3 \mathrm{~K} / \mathrm{Akt}$ could also regulate tumor angiogenesis by promoting endothelial cell migration induced by tumor necrosis factor (TNF). ${ }^{19}$ Furthermore, SEMA3F prevented metastasis of colorectal cancer by PI3K/Akt-dependent down-regulation of the ASCL2/CXCR4 axis. ${ }^{23}$ Ginsenoside Rh2 inhibited metastasis of glioblastoma multiforme through Akt-regulated MMP13. ${ }^{24}$ MiR-218 inhibited the invasion and migration of colon cancer cells by targeting the PI3K/Akt/mTOR signaling pathway. ${ }^{25}$ High glucose promoted tumor invasion and increased metastasis-associated protein expression in human lung epithelial cells by up-regulating HO-1 via reactive oxygen species or the TGF- $\beta 1 /$ PI3K/Akt signaling pathway. ${ }^{26}$

As expected, our investigation revealed that up-regulation of $O$-GlcNAcylation stimulated activation of Akt1 by phosphorylation at Ser473. Moreover, both blocking of PI3K/ Akt pathway by a PI3K-specific inhibitor LY294002 and silencing of Akt1 significantly attenuated the positive regulation of $O$-GlcNAcylation on invasion of thyroid anaplastic cancer cells. These results indicated that PI3K/Akt1 signaling participated in the regulation of $O$-GlcNAcylation on invasion of thyroid anaplastic cancer cells. But which molecules functioned as the targets of $\mathrm{PI} 3 \mathrm{~K} / \mathrm{Akt} 1$ signaling in the regulation process is still unclear. Recently, accumulating evidence demonstrates that $O$-GlcNAcylated target proteins and $O$-GlcNAc-modulated signaling were identified, which were associated with development and progression of tumor. It was reported that the decrease of cell surface E-cadherin and cell adhesion was the molecular mechanism underlying $O$-GlcNAcylation-induced breast cancer metastasis. ${ }^{14}$ Actinbinding protein cofilin was $O$-GlcNAcylated by OGT and mainly, if not completely, mediated OGT modulation of cell mobility. $O$-GlcNAcylation at Ser-108 of cofilin was required for its proper localization in invadopodia at the leading edge of breast cancer cells during three-dimensional cell invasion. ${ }^{27}$ Moreover, we found that the positive impact of $O$-GlcNAcylation on cell invasion had not been completely abolished by LY294002 or Akt1 silencing. These findings implied that there might also be other signaling pathways involved in the regulation of $O$-GlcNAcylation on the invasion of thyroid anaplastic cancer cells simultaneously. Therefore, the molecular mechanism underlying $O$-GlcNAcylation-induced thyroid anaplastic cancer invasion still needs to be further investigated.

A recent study reported that a pyrazolo[3,4- $d]$ pyrimidine compound (CLM3) can inhibit Akt phosphorylation and attenuate the migration and invasion of primary cells from human anaplastic thyroid cancer and $8305 \mathrm{C}$ cells. ${ }^{28}$ Therefore, blocking the PI3K/Akt signaling pathway is a promising approach to the treatment of thyroid cancer. Our study shows that up-regulation of $O$-GlcNAcylation stimulates activation of Akt1 by phosphorylation at Ser473 and enhances the invasion of $8305 \mathrm{C}$ cells. These results suggest that $O$-GlcNAcylation might play a similar role in the primary anaplastic thyroid cancer cells, paving the way to a future clinical evaluation. In order to verify the universality of $O$-GlcNAcylation regulation on the invasion, more thyroid cancer cell lines need to be investigated. In addition, there are at least three Akt family members, Aktl, Akt2, Akt3, which function in a distinct manner in regulating cell migration and invasion. Activity changes of different Akt isoforms and their impacts on the regulation of $O$-GlcNAcylation in cell invasion are still to be addressed.

In conclusion, we present the evidence that $O$-GlcNAcylation promotes the invasion of thyroid anaplastic cancer cells partially dependent on PI3K/Akt1 signaling. It is clear that understanding the underlying molecular mechanism might be significant and beneficial for diagnosis and treatment of cancer.

\section{Disclosure}

The authors report no conflicts of interest in this work. 


\section{References}

1. Katso R, Okkenhaug K, Ahmadi K, White S, Timms J, Waterfield MD. Cellular function of phosphoinositide 3-kinases: implications for development, homeostasis, and cancer. Annu Rev Cell Dev Biol. 2001; 17:615-675.

2. Ward SG, Finan P. Isoform-specific phosphoinositide 3-kinase inhibitors as therapeutic agents. Curr Opin Pharmacol. 2003;3(4):426-434.

3. Solit DB, Basso AD, Olshen AB, Scher HI, Rosen N. Inhibition of heat shock protein 90 function downregulates Akt kinase and sensitizes tumors to Taxol. Cancer Res. 2003;63(9):2139-2144.

4. Arboleda MJ, Lyons JF, Kabbinavar FF, et al. Overexpression of AKT2/protein kinase Bbeta leads to up-regulation of beta1 integrins, increased invasion, and metastasis of human breast and ovarian cancer cells. Cancer Res. 2003;63(1):196-206.

5. Grille SJ, Bellacosa A, Upson J, et al. The protein kinase Akt induces epithelial mesenchymal transition and promotes enhanced motility and invasiveness of squamous cell carcinoma lines. Cancer Res. 2003; 63(9):2172-2178.

6. Comer FI, Hart GW. O-Glycosylation of nuclear and cytosolic proteins. Dynamic interplay between O-GlcNAc and O-phosphate. J Biol Chem. 2000;275(38):29179-29182.

7. Hanover JA, Krause MW, Love DC. The hexosamine signaling pathway: O-GlcNAc cycling in feast or famine. Biochim Biophys Acta. 2010; 1800(2):80-95.

8. Hart GW, Housley MP, Slawson C. Cycling of O-linked $\beta$-Nacetylglucosamine on nucleocytoplasmic proteins. Nature. 2007; 446(7139):1017-1022.

9. Wells L, Vosseller K, Hart GW. Glycosylation of nucleocytoplasmic proteins: signal transduction and O-GlcNAc. Science. 2001; 291(5512):2376-2378.

10. Hanover JA. Glycan-dependent signaling: O-linked N-acetylglucosamine. FASEB J. 2001;15(11):1865-1876.

11. Lazarus BD, Love DC, Hanover JA. O-GlcNAc cycling: implications for neurodegenerative disorders. Int J Biochem Cell Biol. 2009;41(11): 2134-2146.

12. Yang WH, Park SY, Nam HW, et al. NFKB activation is associated with its O-GlcNAcylation state under hyperglycemic conditions. Proc Natl Acad Sci U S A. 2008;105(45):17345-17350.

13. Caldwell SA, Jackson SR, Shahriari KS, et al. Nutrient sensor $\mathrm{O}-\mathrm{GlcNAc}$ transferase regulates breast cancer tumorigenesis through targeting of the oncogenic transcription factor FoxM1. Oncogene. 2010; 29(19):2831-2842.

14. Gu Y, Mi W, Ge Y, et al. GlcNAcylation Plays an Essential Role in Breast Cancer Metastasis. Cancer Res. 2010;70(15):6344-6351.

15. Mi W, Gu Y, Han C, et al. O-GlcNAcylation is a novel regulator of lung and colon cancer malignancy. Biochim Biophys Acta. 2011;1812(4): 514-519.
16. Chang F, Lee JT, Navolanic PM, et al. Involvement of PI3K/Akt pathway in cell cycle progression, apoptosis, and neoplastic transformation: a target for cancer chemotherapy. Leukemia. 2003;17(3):590-603.

17. Lee SH, Kim HS, Park WS, et al. Non-small cell lung cancers frequently express phosphorylated Akt; an immunohistochemical study. APMIS. 2002;110(7-8):587-592.

18. Jeong SJ, Pise-Masison CA, Radonovich MF, Park HU, Brady JN. Activated $\mathrm{AKT}$ regulates $\mathrm{NF} \mathrm{KB}$ activation, $\mathrm{p} 53$ inhibition and cell survival in HTLV-1-transformed cells. Oncogene. 2005;24(44):6719-6728.

19. Zhang R, Xu Y, Ekman N, et al. Etk/Bmx transactivates vascular endothelial growth factor 2 and recruits phosphatidylinositol 3-kinase to mediate the tumor necrosis factorinduced angiogenic pathway. J Biol Chem. 2003;278(51):51267-51276.

20. Namiecinska M, Marciniak K, Nowak JZ. VEGF as an angiogenic, neurotrophic, and neuroprotective factor. Postepy Hig Med Dosw (Online). 2005;59:573-583. Polish.

21. Tan PH, Xue SA, Manunta M, et al. Effect of vectors on human endothelial cell signal transduction. implications for cardiovascular gene therapy. Arterioscler Thromb Vasc Biol. 2006;26(3):462-467.

22. Viglietto G, Motti ML, Bruni P, et al. Cytoplasmic relocalization and inhibition of the cyclin-dependent kinase inhibitor p27(Kip1) by PKB/Akt-mediated phosphorylation in breast cancer. Nat Med. 2002; 8(10):1136-1144.

23. Zhou ZH, Rao J, Wu F, et al. SEMA3F prevented metastasis of colorectal cancer by PI3K/Akt-dependent down-regulation of the ASCL2/ CXCR4 Axis. J Pathol. Epub 2015 Apr 10.

24. Guan N, Huo X, Zhang Z, Zhang S, Luo J, Guo W. Ginsenoside Rh2 inhibited metastasis of glioblastoma multiforme through Akt-regulated MMP13. Tumour Biol. Epub 2015 Apr 3.

25. Zhang X, Shi H, Tang H, Fang Z, Wang J, Cui S. miR-218 inhibited the invasion and migration of colon cancer cells by targeting the PI3K/Akt/ mTOR signaling pathway. Int J Mol Med. 2015;35(5):1301-1308.

26. Kang X, Kong F, Wu X, et al. High glucose promoted tumor invasion and increased metastasis-associated protein expression in human lung epithelial cells by upregulating heme oxygenase- 1 via reactive oxygen species or the TGF- $\beta 1 / \mathrm{PI} 3 \mathrm{~K} /$ Akt signaling pathway. Cell Physiol Biochem. 2015;35(3):1008-1022.

27. Huang X, Pan Q, Sun D, et al. O-GlcNAcylation of cofilin promotes breast cancer cell invasion. J Biol Chem. 2013;288(51):36418-36425.

28. Antonelli A, Bocci G, Fallahi P, et al. CLM3, a multitarget tyrosine kinase inhibitor with antiangiogenic properties, is active against primary anaplastic thyroid cancer in vitro and in vivo. J Clin Endocrinol Metab. 2014;99(4):E572-E581.
OncoTargets and Therapy

\section{Publish your work in this journal}

OncoTargets and Therapy is an international, peer-reviewed, open access journal focusing on the pathological basis of all cancers, potential targets for therapy and treatment protocols employed to improve the management of cancer patients. The journal also focuses on the impact of management programs and new therapeutic agents and protocols on

\section{Dovepress}

patient perspectives such as quality of life, adherence and satisfaction. The manuscript management system is completely online and includes a very quick and fair peer-review system, which is all easy to use. Visit http://www.dovepress.com/testimonials.php to read real quotes from published authors. 